\title{
The effect of tocopherol, vitamin A, selenium and anti-oxidants on ubiquinone in the rat
}

\author{
By A. T. DIPLOCK, E. E. EDWIN, J. BUNYAN AND J. GREEN \\ Walton Oaks Experimental Station, Vitamins Ltd, Tadworth, Surrey
}

(Received I6 fanuary r96I-Revised 27 March г961)

In a previous study (Edwin, Diplock, Bunyan \& Green, I96I), it was shown that administration of vitamin E markedly increased concentrations of ubiquinone in the tissues of the rat. It was found by Heaton, Lowe \& Morton (I957) that ubiquinone concentrations were influenced in the rat by vitamin A status, but Moore \& Sharman (I960) have disputed some of their findings, although they agree that the liver content of ubiquinone is enhanced in vitamin A deficiency, and Green, Edwin, Diplock \& Bunyan (I960) have suggested that at least part of the effect of vitamin A on ubiquinone concentration is due to an indirect action through changes in tocopherol concentrations. Since vitamins $\mathrm{A}$ and $\mathrm{E}$ have a mutually antagonistic action in tissues (Edwin et al. 196I), it was considered important to study the relationship between vitamin $\mathrm{E}$ and ubiquinone in more detail. The previous experiments by Edwin et al. were carried out with rats receiving unusually high amounts of vitamin $A$ in the diet: the experiments reported here were designed to study the effect of vitamin $E$ at various levels of vitamin A nutrition, in order to see whether the relationship was influenced by the amount of vitamin $A$ in the diet.

Several other questions also presented themselves. Edwin et al. have already shown that dietary selenite increases ubiquinone concentrations in the rat, an observation that appears to provide a biochemical connexion for the partial parallelism in the physiological effects of Se and $\alpha$-tocopherol. One aspect requiring further study was whether doses of selenite, administered orally a short time before death, would affect ubiquinone concentrations in the same way as $\alpha$-tocopherol does when so given. Even more important, it was desirable to find out whether Se could influence ubiquinone concentrations in other tissues besides those already examined-especially in the uterus, since Harris, Ludwig \& Schwarz (1958) have demonstrated that Se does not prevent gestation-resorption in the rat. Certain other aspects of the Se effect were also of interest: for example, does supplementation with it increase the tocopherol content of tissues and are both substances necessary for maximal effect on ubiquinone concentrations?

Yet a further subject for study was the effect of anti-oxidants on ubiquinone. Several observations, especially in the chick, have suggested that at least some of the functions of vitamin $\mathrm{E}$ can be duplicated by certain anti-oxidants under physiological conditions, although it is still not clear whether the role of the substances is simply to spare $\alpha$-tocopherol in the tissues: even the excellent study of Draper, Goodyear, Barbee \& Johnson (1958), who showed that diphenyl- $p$-phenylenediamine (DPPD) 
apparently has true vitamin $\mathrm{E}$ activity in the rat, may be vitiated by our finding (Edwin et al. 1961) that the casein used almost certainly contained some $\alpha$-tocopherol. Some workers have suggested that the role of vitamin $\mathrm{E}$ itself may be limited to its function as a tissue anti-oxidant, a view we have already discussed and criticized (Edwin et al. 196r). Preliminary experiments (Green et al. 1960) have indicated that neither DPPD nor Santoquin (1,2-dihydro-6-ethoxy-2,2,4-trimethylquinoline, Monsanto Chemical Co.) is able to affect ubiquinone concentrations in the rat. The action of these substances, particularly the latter, which is believed to be one of the most effective and least toxic of the anti-oxidants in vivo, has been studied in more detail in the series of experiments now described. In addition, we have combined these studies on ubiquinone with a parallel investigation of the peroxidation occurring in certain tissues, using a modification of the thiobarbituric-acid method. We have also studied synthesis of ascorbic acid in some tissues, in view of the demonstration by McCay, Carpenter, Kitabchi \& Caputto (1959) that vitamin E stimulates the synthesis of ascorbic acid in rat-liver homogenates.

\section{EXPERIMENTAL}

Animals and analytical methods. Norwegian hooded rats were used throughout. The methods used for determining tocopherol, ubiquinone, ubichromenol and vitamin A have been previously described (Diplock, Green, Edwin \& Bunyan, I960).

Determination of ascorbic acid. This was done by the 2,4-dinitrophenylhydrazine method of Roe, Mills, Oesterling \& Damron (1948) with the modified reaction conditions $\left(57 \cdot 4^{\circ}\right.$ for $\left.45 \mathrm{~min}\right)$ proposed by Geschwind, Williams \& $\mathrm{Li}$ (195I).

Determination of peroxides. A portion of each tissue was made into a $16 \mathrm{mg} / \mathrm{ml}$ homogenate in ice-cold phosphate buffer $(\mathrm{pH} 7 \cdot 4)$ by means of a nylon-glass homogenizer (taking $30 \mathrm{sec}$ for liver and $60 \mathrm{sec}$ for kidney). Peroxides were determined in vivo after allowing a portion of the cold homogenate to stand at room temperature for to min (see Zalkin \& Tappel, 1960). Peroxidation was studied in vitro by shaking $5 \mathrm{ml}$ of the homogenate at 100 strokes/min for $15 \mathrm{~min}$ in a water-bath at $37^{\circ}$. After addition of $10 \%(\mathrm{w} / \mathrm{v})$ trichloroacetic-acid solution $(5 \mathrm{ml})$ and filtration, a portion of the filtrate was added to an equal volume of $0.5 \%(\mathrm{w} / \mathrm{v})$ thiobarbituric-acid solution in water. The solution was heated in boiling water for $10 \mathrm{~min}$ in a test tube whose mouth was closed with a glass marble. After the solution had cooled in ice-water for $3 \mathrm{~min}$, the optical density of the pink colour was read on an EEL (Evans Electroselenium Ltd) long-cell absorptiometer with an Ilford filter $604\left(\lambda_{\max } 5^{20} \mathrm{~m} \mu\right)$. Results were recorded as readings of the extinction.

Diets. Diets SD I and $\mathrm{A}_{4}$ o have been described by Edwin et al. (1961). Diets Ao and $\mathrm{A}$ ro were similar to diet $\mathrm{A} 4 \mathrm{O}$, but contained, respectively, o and ro i.u. vitamin $\mathrm{A}$ palmitate/g, instead of 40 i.u./g, as in A4o. Diet SAFE had the percentage composition: dried baker's yeast (United Yeast Co. Ltd, Croydon, Surrey) 40, sucrose 40, salt mixture (Edwin et al. 1961) 5, and glucose 15 , and was supplemented with vitamin $D_{3}, \mathrm{r} \cdot 6$ i.u. $/ \mathrm{g}$, and menaphthone, $\mathbf{r} \mu \mathrm{g} / \mathrm{g}$. Diet EroY, a modification of A 10 , contained dried brewer's yeast (Marmite Ltd) 25, casein, 'low vitamin content' 
(Genatosan Ltd) Io, sucrose 59, lard I and McCollum's salt mixture (Edwin et al. 196r) $5 \%$, supplemented with vitamins A and D like diet A ro.

Expt $\mathrm{r}$. Twenty-one groups of female rats, each containing six animals, were reared to IO-I2 weeks of age on diet A Io. Groups I-7 were then given diet Ao, groups 8-I4 diet $\mathrm{A}$ Io, and groups $\mathrm{I}_{5-2 \mathrm{I}}$ diet $\mathrm{A}_{4} \mathrm{O}$, the three diets containing, respectively, $\mathrm{O}$, Io and 40 i.u. vitamin A palmitate/g. They received supplements as follows:

Groups I, 8 and 15 : controls.

Groups 2, 9 and $\mathrm{I} 6$ : two doses of $40 \mathrm{mg}$ Santoquin by mouth 72 and $48 \mathrm{~h}$ before the animals were killed.

Groups 3 , io and 17 : two doses of $5 \mathrm{mg}$ DL- $\alpha$-tocopheryl acetate as above.

Groups 4, II and 18: Santoquin as $0.015 \%(\mathrm{w} / \mathrm{w})$ of the diet.

Groups 5, I2 and 19: Santoquin as $0.1 \%(w / w)$ of the diet.

Groups 6, I3 and 20: $\alpha$-tocopheryl acetate as $0.01 \%(w / w)$ of the diet.

Groups 7, I4 and 21: sodium selenite in the diet to give o.I p.p.m. Se.

After 6 weeks on the diets, the animals were killed with ether. Heart, liver, lungs, uterus and kidneys were analysed by the methods previously described (Diplock et al. I960). In this method, preliminary purification of extracts is carried out by chromatography on Decalso F (Permutit Co., London) and elution with benzene. It has become apparent that Decalso $F$, although a synthetic absorbent, is liable to considerable variation. Its bulk density can vary from 0.68 to 0.88 and cannot be readily controlled by the manufacturers. The absorptive properties vary with the density and $35 \mathrm{ml}$ of benzene may not be sufficient to elute quantitatively the tocopherols and ubiquinone (especially the latter). In most experiments with the more absorptive material, $5^{\circ} \mathrm{ml}$ of benzene has been found suitable to elute normal extracts, but it is recommended that Decalso $\mathrm{F}$ be checked for quantitative elution before use. In more recent work it has been found that $30 \mathrm{ml}$ of a $35 \%(\mathrm{v} / \mathrm{v})$ solution of diethyl ether in light petroleum (b.p. $40-60^{\circ}$ ) is an excellent eluant for $\alpha$-tocopherol and ubiquinones, and this solvent mixture possesses the advantage of being easier to deal with in the subsequent evaporation. The volume of solvent should not exceed $30 \mathrm{ml}$ (for the standard-size columns used) or undesirable impurities may be eluted.

The liver and kidneys of one rat from each of nineteen groups were examined for peroxides, peroxide formation and concentrations of ascorbic acid, this being all that was feasible because of the time relationships of the experiment.

Expt 2. It was planned to rear very young rats on a diet more rigorously freed of vitamin $\mathrm{E}$ than hitherto attempted, and to study the effects of vitamin $\mathrm{E}$ and other substances on the tissues of young animals. Diet SAFE was used, casein and lard being eliminated, since they have both been found to contain appreciable amounts of $\alpha$-tocopherol. The diet was based on a dried baker's yeast containing very little tocopherol (Diplock, Green, Edwin \& Bunyan, unpublished results) and known to be deficient in Se.

One hundred and sixty-five rats (ninety-three males and seventy-two females) were divided into eleven groups, containing approximately equal numbers of each sex. Males and females of the litters available were randomized over the eleven groups. The animals were given diet SAFE supplemented with O.I p.p.m. Se and 3 i.u. 
vitamin A/g from the age of 13 to 28 days. They were then given diet SAFE without the supplements and received various treatments as follows:

Group I: controls.

Group 2: $3.5 \mathrm{mg} \alpha$-tocopheryl acetate by mouth $24 \mathrm{~h}$ before the animals were killed.

Group 3: rooo i.u. vitamin A palmitate as above.

Group 4:2 $\mu \mathrm{g}$ Se as an aqueous solution of sodium selenite as above.

Group 5: 40 i.u. vitamin A palmitate/g diet.

Group 6:0.1 $\mathrm{mg} \alpha$-tocopheryl acetate/g diet.

Groups 7,8, 9, Io and II : sodium selenite in the diet to give O.I p.p.m. Se, and various supplements as follows:

Group 7: controls given Se.

Group 8:3.5 mg of $\alpha$-tocopheryl acetate by mouth $24 \mathrm{~h}$ before the animals were killed.

Group 9: I000 i.u. vitamin A palmitate as above.

Group Io: 40 i.u. vitamin A palmitate/g diet.

Group I I : $0.1 \mathrm{mg} \alpha$-tocopheryl acetate/g diet.

Many deaths occurred on diet SAFE within the Ist fortnight. At autopsy, many of the rats showed typical signs of necrotic liver degeneration, even at this age. The experiment was ended after 3 weeks, when the rats were killed with ether, and hearts and livers from equal numbers of each sex remaining were taken for analysis.

Expt 3. Three groups of eight female rats were given diet A ro from the age of I3 days until they were 4 months old. The diet of one group was then supplemented with $0.05 \%(\mathrm{w} / \mathrm{w})$ of DPPD and that of the second with $0.10 \%$ of Santoquin; the third group continued on the unsupplemented diet. After 3 weeks, the animals were killed by breaking their necks, and hearts and livers in each group were analysed for the usual lipid constituents. Their livers were examined, in addition, for 'in vivo' peroxides and 'in vitro' peroxidation. 'Zero time' thiobarbituric-acid analyses were carried out besides the normal ro min test. The livers were also analysed for vitamin A to study the protective effects of the anti-oxidants.

Expt 4. Two groups of six female rats, aged 4 months, were selected at random from the stock colony. One group continued to receive stock diet SDI and the other received a dietary supplement of $0 . \mathrm{I}$ p.p.m. Se (as sodium selenite). After $4 \mathrm{I}$ days, the animals were killed with ether, and hearts and livers were analysed.

Expt 5. Three groups of ten female rats were reared on the vitamin E-deficient diet $\mathrm{E}_{\mathrm{I}} \mathrm{OY}$ from the age of $\mathrm{I} 3$ days until they were 6 months old. One group was given by mouth doses of $5 \mathrm{mg} \alpha$-tocopheryl acetate 72 and $48 \mathrm{~h}$ before slaughter. The second group received by mouth two doses of Io $\mu \mathrm{g}$ Se (as sodium selenite) at the same time. The third group acted as controls. The animals were killed by breaking their necks, and ovaries, uteri and pituitaries were taken for analysis. 


\section{RESULTS}

Expt $\mathrm{I}$. The aim of this experiment, the results of which are given in Tables $\mathrm{I}-8$, was to compare the concentrations of ubiquinone when different amounts of vitamin $\mathrm{A}$ were given in the diet and also to study the effect of vitamin $\mathrm{E}, \mathrm{Se}$ and Santoquin on ubiquinone concentration when the amount of vitamin $\mathrm{A}$ in the diet was varied. There was no attempt to produce complete vitamin A deficiency, which introduces another variable, whose nature still remains unresolved (see Moore \& Sharman, 1960).

\section{Table I. Expt I. Effect of vitamin E, vitamin $A$, Santoquin and selenium on ubiquinone and ubichromenol concentration in rat heart}

(Mean values for groups of six female rats. Groups $1-7$ were on diet Ao, 8-14 on diet $A_{10}$ and $r_{5}-21$ on $\operatorname{diet} A_{4}$, see p. 426)

\begin{tabular}{|c|c|c|c|c|c|c|}
\hline $\begin{array}{c}\text { Group } \\
\text { no. }\end{array}$ & $\begin{array}{l}\text { Treatment } \\
\text { (see p. } 427)\end{array}$ & $\begin{array}{l}\text { Heart weight } \\
\text { (g) }\end{array}$ & $\begin{array}{l}\text { Ubi- } \\
\text { quinone } \\
(\mu \mathrm{g} / \mathrm{g})\end{array}$ & $\begin{array}{c}\text { Ubi- } \\
\text { chromenol } \\
(\mu \mathrm{g} / \mathrm{g})\end{array}$ & $\begin{array}{c}\alpha \text {-Toco- } \\
\text { pherol } \\
(\mu \mathrm{g} / \mathrm{g})\end{array}$ & $\begin{array}{l}\text { Vitamin } A \\
\quad \text { (i.u./g) }\end{array}$ \\
\hline $\mathbf{I}$ & A. None & 0.762 & 142 & 9 & $7 \cdot 7$ & $2 \cdot 9$ \\
\hline 2 & B. Santoquin, final dose & 0.882 & I6I & 12 & $7 \cdot 4$ & $2 \cdot 9$ \\
\hline 3 & C. $\alpha$-Tocopherol, final dose & 0.920 & $15 \mathrm{I}$ & 12 & $17 \cdot 0$ & $3 \cdot 7$ \\
\hline 4 & D. Santoquin, $0.015 \%$ of diet & 0.864 & 162 & I I & $6 \cdot 3$ & $3 \cdot 0$ \\
\hline 5 & E. Santoquin, $0.1 \%$ of diet & 0.829 & 163 & Io & $5 \cdot 9$ & $2 \cdot 5$ \\
\hline 6 & F. $\alpha$-Tocopherol, $0^{\circ} \circ \mathrm{r} \%$ of diet & t $\quad 0.782$ & 198 & 13 & $5 I \cdot 8$ & 3.7 \\
\hline 7 & G. Se, 0.1 p.p.m. in diet & 0.779 & 200 & I3 & $6 \cdot 5$ & $3 \cdot 6$ \\
\hline 8 & A. None & 0.805 & 142 & 7 & $6 \cdot 0$ & $3 \cdot 5$ \\
\hline 9 & B. Santoquin, final dose & 0.873 & 151 & 8 & 5.5 & $3 \cdot 2$ \\
\hline Io & C. $\alpha$-Tocopherol, final dose & 0.930 & 202 & 10 & 14.8 & $3 \cdot 1$ \\
\hline Ir & D. Santoquin, $0.015 \%$ of diet & 0.849 & 144 & 8 & $3 \cdot 8$ & $3 \cdot 3$ \\
\hline 12 & E. Santoquin, $0.1 \%$ of diet & 0.821 & 144 & 8 & $4 \cdot 3$ & $3 \cdot 2$ \\
\hline 13 & F. $\alpha$-Tocopherol, o.or $\%$ of diet & 0.819 & 184 & 10 & $39^{\circ} 8$ & $3 \cdot 1$ \\
\hline 14 & G. Se, o. I p.p.m. in diet & 0.816 & 180 & 7 & $3 \cdot 2$ & $3 \cdot 1$ \\
\hline $\mathbf{I} 5$ & A. None & 0.843 & I 44 & 7 & $\mathrm{I} \cdot 8$ & $3 \cdot \mathbf{I}$ \\
\hline 16 & B. Santoquin, final dose & 0.874 & I 5 I & 7 & $1 \cdot 3$ & 3.4 \\
\hline I7 & C. $\alpha$-Tocopherol, final dose & 0.827 & 165 & 9 & $12 \cdot 6$ & $4 \cdot I$ \\
\hline 18 & D. Santoquin, $0.015 \%$ of diet & 0.807 & 133 & 7 & $\mathbf{I} \cdot 3$ & $3 \cdot 2$ \\
\hline 19 & E. Santoquin, $0.1 \%$ of diet & 0.803 & 128 & 7 & $\mathrm{I} \cdot 4$ & $3 \cdot 2$ \\
\hline 20 & F. $\alpha$-Tocopherol, $0.01 \%$ of diet & 0.842 & 170 & 9 & $44 \cdot 5$ & $3 \cdot 8$ \\
\hline $2 \mathrm{I}$ & G. Se, o.I p.p.m. in diet & 0.763 & 195 & 12 & $3 \cdot 2$ & $3 \cdot 4$ \\
\hline
\end{tabular}

The results for five tissues (heart, liver, kidney, lung and uterus) are given in Tables I-5. In order to assess the effect of the different treatments, two additional tables have been prepared and these present, first, the mean results for treatment effects regardless of dietary supplementation with vitamin A (Table 6) and, secondly, the mean results for the effects of dietary vitamin A regardless of the other treatments (Table 7). Organ weights and ubichromenol concentrations appeared to be of less interest and are not summarized in Tables 6 and 7 . The variance of the concentrations of ubiquinone, $\alpha$-tocopherol and vitamin $A$ in each tissue was analysed in the usual way, the treatment $\times$ vitamin A level interaction being used as an estimate of error (its significance could not be tested because duplicate determinations were not carried out). The results of the statistical analyses are presented in Tables 6 and 7 .

Table I summarizes the results for rat heart; little effect of vitamin A supplementation on ubiquinone concentration or even on vitamin $A$ concentration can be noted, 
Table 2. Expt I. Effect of vitamin $E$, vitamin $A$, Santoquin and selenium on ubiquinone and ubichromenol concentration in rat liver

(Mean values for groups of six female rats. Groups $I-7$ were on $\operatorname{diet}$ Ao, 8-I4 on $\operatorname{diet} \mathrm{A}_{10} \mathrm{and}$ I $_{5}-2 \mathrm{I}$ on $\operatorname{diet} \mathrm{A} 40$, see p. 426)

\begin{tabular}{|c|c|c|c|c|c|c|}
\hline $\begin{array}{l}\text { Group } \\
\text { no. }\end{array}$ & $\begin{array}{l}\text { Treatment } \\
\text { (see p. 427) }\end{array}$ & $\begin{array}{l}\text { Heart weight } \\
\text { (g) }\end{array}$ & $\begin{array}{l}\text { Ubi- } \\
\text { quinone } \\
(\mu \mathrm{g} / \mathrm{g})\end{array}$ & $\begin{array}{c}\text { Ubi- } \\
\text { chromenol } \\
(\mu \mathrm{g} / \mathrm{g})\end{array}$ & $\begin{array}{c}\alpha \text {-Toco- } \\
\text { pherol } \\
(\mu \mathrm{g} / \mathrm{g})\end{array}$ & $\begin{array}{c}\text { Vitamin } A \\
\text { (i.u./g) }\end{array}$ \\
\hline I & A. None & $9 \cdot 3^{8}$ & 95 & 62 & 5.5 & 15 \\
\hline 2 & B. Santoquin, final dose & $10 \cdot 21$ & 97 & $6 r$ & $6 \cdot 6$ & 17 \\
\hline 3 & C. $\alpha$-Tocopherol, final dose & $10 \cdot 33$ & I 29 & 50 & $28 \cdot 5$ & 17 \\
\hline 4 & D. Santoquin, $0.015 \%$ of diet & $9 \cdot$ II & IIO & $7 \mathrm{I}$ & $8 \cdot 3$ & 12 \\
\hline 5 & E. Santoquin, $\alpha \cdot I \%$ of diet & 10.40 & 93 & 62 & 5.0 & 8 \\
\hline 6 & F. $\alpha$-Tocopherol, $0.01 \%$ of diet & $9 \cdot 23$ & II I & 85 & $4 I \cdot 6$ & 17 \\
\hline 7 & G. Se, o.I p.p.m. in diet & $9 \cdot 36$ & I I I & 77 & 3.5 & 16 \\
\hline 8 & A. None & $9 \cdot 23$ & $5^{8}$ & 39 & $1 \cdot 7$ & 222 \\
\hline 9 & B. Santoquin, final dose & $9 \cdot 99$ & 73 & 54 & 10.5 & 238 \\
\hline ro & C. $\alpha$-Tocopherol, final dose & 10.03 & 93 & 66 & 23.0 & 248 \\
\hline II & D. Santoquin, $0.015 \%$ of diet & 10.00 & 62 & 39 & $5 \cdot 0$ & 172 \\
\hline 12 & E. Santoquin, $O .1 \%$ of diet & $10 \cdot 26$ & $5 \mathrm{I}$ & 36 & $6 \cdot 3$ & 150 \\
\hline 13 & F. $\alpha$-Tocopherol, $0.01 \%$ of diet & $9 \cdot 47$ & 105 & 59 & $39 \cdot 6$ & 247 \\
\hline 14 & G. Se, o.I p.p.m. in diet & $10 \cdot 29$ & 74 & 42 & $6 \cdot 3$ & 223 \\
\hline 15 & A. None & $8 \cdot 66$ & 62 & 47 & 0.7 & 232 \\
\hline 16 & B. Santoquin, final dose & $10 \cdot 36$ & 65 & 50 & $6 \cdot 6$ & 238 \\
\hline 17 & C. $\alpha$-Tocopherol, final dose & $9 \cdot 17$ & 83 & 45 & $30 \cdot 0$ & 328 \\
\hline 18 & D. Santoquin, $0.015 \%$ of diet & $8 \cdot 23$ & 65 & 50 & $3 \cdot 3$ & 220 \\
\hline I9 & E. Santoquin, $0.1 \%$ of diet & I 188 & 68 & 45 & $6 \cdot 5$ & 242 \\
\hline 20 & F. $\alpha$-Tocopherol, $0.01 \%$ of diet & t $\quad 10.88$ & 92 & 57 & $4 \mathrm{I} \cdot 9$ & 306 \\
\hline $2 \mathrm{I}$ & G. Se, O.I p.p.m. in diet & $9 \cdot 83$ & 93 & 63 & $6 \cdot 3$ & 250 \\
\hline
\end{tabular}

Table 3. Expt r. Effect of vitamin $E$, vitamin $A$, Santoquin and selenium on ubiquinone and ubichromenol concentration in rat kidney

\begin{tabular}{|c|c|c|c|c|c|c|}
\hline $\begin{array}{c}\text { Group } \\
\text { no. }\end{array}$ & $\begin{array}{l}\text { Treatment } \\
\text { (see p. 427) }\end{array}$ & $\begin{array}{l}\text { Heart weight } \\
\text { (g) }\end{array}$ & $\begin{array}{l}\text { Ubi- } \\
\text { quinone } \\
(\mu \mathrm{g} / \mathrm{g})\end{array}$ & $\begin{array}{l}\text { Ubi- } \\
\text { chromenol } \\
(\mu \mathrm{g} / \mathrm{g})\end{array}$ & $\begin{array}{c}\alpha \text {-Toco- } \\
\text { pherol } \\
(\mu \mathrm{g} / \mathrm{g})\end{array}$ & $\begin{array}{l}\text { Vitamin A } \\
\text { (i.u./g) }\end{array}$ \\
\hline I & A. None & $I \cdot 64$ & 76 & 7 & $2 \cdot 9$ & $3 \cdot 6$ \\
\hline 2 & B. Santoquin, final dose & $I \cdot 69$ & 73 & 7 & 3.0 & $3 \cdot 0$ \\
\hline 3 & C. $\alpha$-Tocopherol, final dose & $I \cdot 76$ & 92 & 6 & $12 \cdot 9$ & 4.7 \\
\hline 4 & D. Santoquin, $0.015 \%$ of diet & $x \cdot 66$ & 78 & 7 & $3 \cdot 6$ & $3 \cdot 4$ \\
\hline 5 & E. Santoquin, $0.1 \%$ of diet & I.79 & 74 & 8 & $4^{\cdot} \cdot \mathrm{I}$ & $2 \cdot 9$ \\
\hline 6 & F. $\alpha$-Tocopherol, $0.01 \%$ of diet & $I \cdot 59$ & 89 & 8 & $32 \cdot 4$ & $5 \cdot 3$ \\
\hline 7 & G. Se, 0.1 p.p.m. in diet & $1 \cdot 60$ & $9 I$ & 5 & 3.7 & $3 \cdot 6$ \\
\hline 8 & A. None & $I \cdot 64$ & 84 & 5 & 3.5 & $3 \cdot 8$ \\
\hline 9 & B. Santoquin, final dose & $1 \cdot 69$ & 80 & 4 & 4.1 & $3 \cdot 8$ \\
\hline 10 & C. $\alpha$-Tocopherol, final dose & $I \cdot 84$ & 93 & 7 & $12 \cdot 6$ & $4 \cdot 0$ \\
\hline I I & D. Santoquin, $0.015 \%$ of diet & $x \cdot 73$ & 71 & 4 & $4 \cdot 2$ & $3 \cdot 2$ \\
\hline I 2 & E. Santoquin, $0.1 \%$ of diet & $1 \cdot 71$ & 74 & 5 & $4 \cdot 9$ & $3 \cdot 1$ \\
\hline I3 & F. $\alpha$-Tocopherol, 0.0 I $\%$ of diet & $r \cdot 68$ & 90 & 9 & $28 \cdot 8$ & 4.9 \\
\hline 14 & G. Se, $0^{\prime}$ p p.p.m. in diet & $I \cdot 6 I$ & $9 \mathrm{I}$ & 9 & $3 \cdot 8$ & $3 \cdot 2$ \\
\hline I 5 & A. None & $I \cdot 62$ & 56 & 4 & $I \cdot 9$ & $3 \cdot 3$ \\
\hline I6 & B. Santoquin, final dose & $\mathbf{I} \cdot 8 \mathbf{5}$ & 59 & 7 & 4.0 & $2 \cdot 8$ \\
\hline I 7 & C. $\alpha$-Tocopherol, final dose & $1 \cdot 71$ & 79 & 5 & II. 4 & $4 \cdot 4$ \\
\hline I8 & D. Santoquin, $0.015 \%$ of diet & $\mathbf{r} \cdot 63$ & 44 & 4 & 3.5 & $3 \cdot 5$ \\
\hline 19 & E. Santoquin, $0.1 \%$ of diet & $x \cdot 75$ & 50 & 5 & $5^{\circ} \mathrm{O}$ & $3 \cdot 1$ \\
\hline 20 & F. $\alpha$-Tocopherol, $0.01 \%$ of diet & $I \cdot 77$ & 66 & 8 & $14 \cdot 9$ & $5 \cdot 6$ \\
\hline $2 \mathrm{I}$ & G. Se, 0.1 p.p.m. in diet & $I \cdot 71$ & 70 & 7 & $4 \cdot 8$ & $3 \cdot 6$ \\
\hline
\end{tabular}


but Table 7 shows a significant reduction of $\alpha$-tocopherol content with increasing content of vitamin A in the diet. Tocopherol (by mouth and in the diet) and Se significantly raised ubiquinone concentration (Table 6).

Table 2 gives the results for liver. The effect of the increased vitamin A contents in the groups on diets $\mathrm{A}_{\text {ro }}$ and $\mathrm{A}_{4} \mathrm{O}$ was to depress ubiquinone concentration in liver, without having a significant effect on tocopherol concentration. Ubiquinone concentration was increased significantly by tocopherol or Se and this effect occurred irrespective of the vitamin A level. Santoquin was without significant effect on ubiquinone concentration. The results in Table 2 tend to support our earlier conclusion that in liver there is a vitamin A effect in depressing ubiquinone content and it may be superimposed on the simultaneous effect due to vitamin $\mathrm{E}$.

\section{Table 4. Expt 1. Effect of vitamin E, vitamin A, Santoquin and selenium on ubiquinone and ubichromenol concentration in rat lung}

(Mean values for groups of six female rats. Groups $\mathrm{I}-7$ were on $\operatorname{diet} \mathrm{Ao}$, 8-I4 on diet $A_{10}$ and $I_{5-21}$ on $\operatorname{diet} A_{40}$, see p. 426)

\begin{tabular}{|c|c|c|c|c|c|}
\hline $\begin{array}{c}\text { Group } \\
\text { no. }\end{array}$ & $\begin{array}{l}\text { Treatment } \\
\text { (see p. 427) }\end{array}$ & $\begin{array}{l}\text { rt weight } \\
\text { (g) }\end{array}$ & $\begin{array}{l}\text { Ubiquinone } \\
\qquad(\mu \mathrm{g} / \mathrm{g})\end{array}$ & $\begin{array}{c}\alpha-\text { Tocopherol } \\
(\mu \mathrm{g} / \mathrm{g})\end{array}$ & $\begin{array}{l}\text { Vitamin A } \\
\text { (i.u./g) }\end{array}$ \\
\hline $\mathbf{I}$ & A. None & $I \cdot 56$ & 12 & $4 \cdot 8$ & 4.4 \\
\hline 2 & B. Santoquin, final dose & $\mathrm{I} \cdot 86$ & 14 & $4 \cdot 8$ & $5 \cdot 2$ \\
\hline 3 & C. $\alpha$-Tocopherol, final dose & $1 \cdot 68$ & 22 & $23 \cdot 2$ & $6 \cdot 4$ \\
\hline 4 & D. Santoquin, $0.015 \%$ of diet & $1 \cdot 92$ & 13 & $3 \cdot 9$ & $3 \cdot 0$ \\
\hline 5 & E. Santoquin, $0.1 \%$ of diet & $I \cdot 60$ & 10 & 44 & $3 \cdot 3$ \\
\hline 6 & F. $\alpha$-Tocopherol, $0.01 \%$ of diet & $1 \cdot 53$ & 19 & $46 \cdot 4$ & $4 \cdot 8$ \\
\hline 7 & G. Se, 0.1 p.p.m. in diet & $1 \cdot 50$ & 17 & $4 \cdot \mathrm{I}$ & $3 \cdot 9$ \\
\hline 8 & A. None & $\mathbf{I} \cdot 70$ & Io & $2 \cdot 5$ & 5.9 \\
\hline 9 & B. Santoquin, fnal dose & I 49 & I I & $3 \cdot 2$ & - \\
\hline I0 & C. $\alpha$-Tocopherol, final dose & $1 \cdot 72$ & 14 & 20.4 & - \\
\hline II & D. Santoquin, $0.015 \%$ of diet & $I \cdot 5 I$ & 12 & $3 \cdot 7$ & - \\
\hline 12 & E. Santoquin, $0.1 \%$ of diet & $I \cdot 38$ & 10 & $4 \cdot I$ & 一 \\
\hline I3 & F. $\alpha$-Tocopherol, $0.01 \%$ of diet & $I \cdot 66$ & 13 & $34 \cdot 2$ & $6 \cdot 2$ \\
\hline 14 & G. Se, 0.1 p.p.m. in diet & $I \cdot 55$ & I3 & $1 \cdot 9$ & $8 \cdot 2$ \\
\hline I 5 & A. None & $I \cdot 48$ & 10 & $I \cdot 9$ & $10 \cdot 3$ \\
\hline 16 & B. Santoquin, final dose & $I \cdot 66$ & I I & $2 \cdot 3$ & $14 \cdot 2$ \\
\hline 17 & C. $\alpha$-Tocopherol, final dose & $1 \cdot 53$ & 15 & $17 \cdot 8$ & 10.0 \\
\hline 18 & D. Santoquin, $0.015 \%$ of diet & $1 \cdot 63$ & 9 & $2 \cdot 4$ & $10 \cdot 6$ \\
\hline 19 & E. Santoquin, $0.1 \%$ of diet & $x \cdot 67$ & 9 & $2 \cdot 8$ & I $I \cdot 2$ \\
\hline 20 & F. $\alpha$-Tocopherol, $0.01 \%$ of diet & $I \cdot 6 I$ & 19 & $35^{\cdot 8}$ & $12 * 4$ \\
\hline $2 \mathrm{I}$ & G. Se, o.1 p.p.m. in diet & $x \cdot 83$ & I6 & $x \cdot 8$ & $11 \cdot 0$ \\
\hline
\end{tabular}

No ubichromenol was detected in any sample.

Table 3 gives the results for kidney. In this tissue, increased dietary supplementation with vitamin A had no effect on either vitamin A or tocopherol concentration. The decrease in ubiquinone concentration in the kidneys of the $\mathrm{A}_{40}$ group was associated with a lowered vitamin $\mathrm{E}$ concentration in the tissue and not with an increased vitamin A concentration. Under all three conditions of vitamin A nutrition, however, $\alpha$-tocopherol, whether given by mouth as a dose just before death or as a dietary supplement, increased ubiquinone concentration. Santoquin significantly depressed the ubiquinone concentration.

Table 4 gives the results for lung. In this tissue vitamin A concentration increased 
'Table 5. Expt I. Effect of vitamin E, vitamin A, Santoquin and selenium on ubiquinone and ubichromenol concentration in rat uterus*

(Mean values for groups of six female rats. Groups I-7 were on diet Ao, 8-14 on diet $A_{10}$ and I $^{-2}$ I on diet A 40 , see p. 426)

\begin{tabular}{|c|c|c|c|c|c|}
\hline $\begin{array}{l}\text { Group } \\
\text { no. }\end{array}$ & $\begin{array}{l}\text { Treatment } \\
\text { (see p. } 427)\end{array}$ & $\begin{array}{l}\text { Ieart weight } \\
\text { (g) }\end{array}$ & $\begin{array}{l}\text { Ubiquinone } \\
\qquad(\mu \mathrm{g} / \mathrm{g})\end{array}$ & $\begin{array}{l}\text { Ubichromenol } \\
\qquad(\mu \mathrm{g} / \mathrm{g})\end{array}$ & $\begin{array}{c}\alpha-\text { Tocopherol } \\
(\mu \mathrm{g} / \mathrm{g})\end{array}$ \\
\hline I & A. None & 0.828 & 12 & 3 & $4^{*} 5$ \\
\hline 2 & B. Santoquin, final dose & 0.759 & 15 & 3 & $8 \cdot 0$ \\
\hline 3 & C. $\alpha$-'Tocopherol, final dose & I.097 & 17 & 2 & $43 \cdot 8$ \\
\hline 4 & D. Santoquin, $0.015 \%$ of diet & 0.794 & I4 & 4 & $5 \cdot 4$ \\
\hline 5 & E. Santoquin, $\mathrm{o} . \mathrm{I} \%$ of diet & 0.693 & - & - & - \\
\hline 6 & F. $\alpha$-Tocopherol, $0.01 \%$ of diet & 0.696 & 18 & 5 & $69^{\circ} \circ$ \\
\hline 7 & G. Se, o.I p.p.m. in diet & $0.73^{\circ}$ & 12 & 4 & $3 \cdot 8$ \\
\hline 8 & A. None & 0.894 & I I & 5 & $2 \cdot 8$ \\
\hline 9 & B. Santoquin, final dose & 0.716 & I5 & 4 & $7 \cdot 2$ \\
\hline 10 & C. $\alpha$-Tocopherol, final dose & 0.860 & 17 & 3 & $47 \cdot 6$ \\
\hline I I & D. Santoquin, o.0 $5 \%$ of diet & 0.827 & 15 & 3 & $5 \cdot 6$ \\
\hline 12 & E. Santoquin, $0.1 \%$ of diet & 0.792 & 13 & 4 & $8 \cdot 9$ \\
\hline 13 & F. $\alpha$-Tocopherol, $0.01 \%$ of diet & 0.950 & 18 & 3 & $75 \cdot 9$ \\
\hline 14 & G. Se, o. I p.p.m. in diet & $0.78 \mathrm{I}$ & I I & 4 & $6 \cdot 2$ \\
\hline 15 & A. None & 0.796 & 13 & 3 & $3 \cdot 2$ \\
\hline 16 & B. Santoquin, final dose & $0.93 I$ & 14 & 3 & $8 \cdot 2$ \\
\hline 17 & C. $\alpha$-Tocopherol, final dose & 0.800 & I 5 & 4 & $24 \cdot 8$ \\
\hline ז8 & D. Santoquin, $0.015 \%$ of diet & 0.664 & ro & 6 & $5 \cdot 7$ \\
\hline 19 & E. Santoquin, $\circ .1 \%$ of diet & 0.635 & 23 & 6 & 10.7 \\
\hline 20 & F. $\alpha$-Tocopherol, $0.01 \%$ of diet & $t 0.663$ & 22 & 6 & $72 \cdot 4$ \\
\hline 21 & G. Se, O.I p.p.m. in diet & 0.767 & 12 & 4 & $9 \cdot 9$ \\
\hline
\end{tabular}

The amounts of tissues were too small for estimation of vitamin A.

* Including ovary and fallopian tube.

\section{Table 6. Expt I. Effect of six treatments on tissue concentrations of ubiquinone, $\alpha$-tocopherol and vitamin $A$ in the rat}

(Each value is the mean of the treatment results given in Tables $1-5$, regardless of the dietary level of vitamin A)

\begin{tabular}{|c|c|c|c|c|c|c|c|c|}
\hline \multirow[b]{2}{*}{ Tissue } & \multirow[b]{2}{*}{ Parameter } & \multicolumn{7}{|c|}{ Treatment } \\
\hline & & A & B & $\mathrm{C}$ & D & $\mathbf{E}$ & $\mathbf{F}$ & $\mathrm{G}$ \\
\hline Heart & $\begin{array}{l}\text { Ubiquinone }(\mu \mathrm{g} / \mathrm{g}) \\
\alpha \text {-Tocopherol }(\mu \mathrm{g} / \mathrm{g}) \\
\text { Vitamin A (i.u. } / \mathrm{g})\end{array}$ & $\begin{array}{r}143 \\
5 \cdot 2 \\
3 \cdot 2\end{array}$ & $\begin{array}{r}154 \\
4 \cdot 7 \\
3 \cdot 2\end{array}$ & $\begin{array}{l}\text { I } 73^{* * *} \\
14 \cdot 8^{* *} \\
3 \cdot 6\end{array}$ & $\begin{array}{l}146 \\
3 \cdot 8 \\
3 \cdot 2\end{array}$ & $\begin{array}{l}145 \\
3 \cdot 9 \\
3 \cdot 0\end{array}$ & $\begin{array}{l}184^{* *} \\
45.4 * \\
3.5\end{array}$ & $\begin{array}{l}192^{* * *} \\
4 * 3 \\
3 \cdot 4\end{array}$ \\
\hline Liver & $\begin{array}{l}\text { Ubiquinone }(\mu \mathrm{g} / \mathrm{g}) \\
\alpha \text {-Tocopherol }(\mu \mathrm{g} / \mathrm{g}) \\
\text { Vitamin A (i.u. } / \mathrm{g})\end{array}$ & $\begin{array}{r}72 \\
2 \cdot 6 \\
156\end{array}$ & $\begin{array}{c}78 \\
7 \cdot 9^{*} \\
164\end{array}$ & $\begin{array}{l}102^{* *} \\
27 \cdot 2^{* * *} \\
198\end{array}$ & $\begin{array}{r}79 \\
5.5 \\
\times 35\end{array}$ & $\begin{array}{r}71 \\
5 \cdot 9 \\
133\end{array}$ & $\begin{array}{l}103 * * \\
41 \cdot 0^{* * *} \\
190\end{array}$ & $\begin{array}{c}93^{*} \\
5 \cdot 4 \\
163\end{array}$ \\
\hline Kidney & $\begin{array}{l}\text { Ubiquinone }(\mu \mathrm{g} / \mathrm{g}) \\
\alpha \text {-Tocopherol }(\mu \mathrm{g} / \mathrm{g}) \\
\text { Vitamin A (i.u. } / \mathrm{g})\end{array}$ & $\begin{array}{r}72 \\
2 \cdot 8 \\
3 \cdot 6\end{array}$ & $\begin{array}{r}71 \\
3 \cdot 7 \\
3 \cdot 2\end{array}$ & $\begin{array}{r}88^{* * *} \\
\text { I } 2 \cdot 3^{* *} \\
4 \cdot 4^{*}\end{array}$ & $\begin{array}{l}64^{*} \\
3 \cdot 8 \\
3 \cdot 4\end{array}$ & $\begin{array}{c}66 \\
4 \cdot 7 \\
3^{\cdot} 0^{*}\end{array}$ & $\begin{array}{r}82^{* * *} \\
25 \cdot 4^{* *} \\
5 \cdot 3^{* *}\end{array}$ & $\begin{array}{l}84^{* * *} \\
4 \cdot 1 \\
3 \cdot 5\end{array}$ \\
\hline Lung & $\begin{array}{l}\text { Ubiquinone }(\mu \mathrm{g} / \mathrm{g}) \\
\alpha \text {-Tocopherol }(\mu \mathrm{g} / \mathrm{g}) \\
\text { Vitamin A (i.u. } / \mathrm{g})\end{array}$ & $\begin{array}{r}11 \\
3 \cdot 1 \\
6 \cdot 9\end{array}$ & $\begin{array}{r}12 \\
3 \cdot 4 \\
9 \cdot 7\end{array}$ & $\begin{array}{c}17^{* * *} \\
20 \cdot 5^{* * *} \\
8 \cdot 2\end{array}$ & $\begin{array}{r}\text { I I } \\
3 \cdot 3 \\
6 \cdot 8\end{array}$ & $\begin{array}{r}10 \\
3 \cdot 8 \\
7 \cdot 2\end{array}$ & $\begin{array}{c}17^{* *} \\
38 \cdot 8^{* *} \\
7 \cdot 8\end{array}$ & $\begin{array}{l}15^{*} \\
2 \cdot 6 \\
7 \cdot 7\end{array}$ \\
\hline Uterus & $\begin{array}{l}\text { Ubiquinone }(\mu \mathrm{g} / \mathrm{g}) \\
\alpha \text {-Tocopherol }(\mu \mathrm{g} / \mathrm{g})\end{array}$ & $\begin{array}{r}12 \\
3 \cdot 5\end{array}$ & $\begin{array}{r}15 \\
7 \cdot 8\end{array}$ & $\begin{array}{c}16^{*} \\
38 \cdot 7^{* *}\end{array}$ & $\begin{array}{r}13 \\
5 \cdot 6\end{array}$ & $\begin{array}{r}18 \\
9 \cdot 8\end{array}$ & $\begin{array}{r}19^{* *} \\
72 * 4^{* *}\end{array}$ & $\begin{array}{r}12 \\
6 \cdot 6\end{array}$ \\
\hline
\end{tabular}

* Significantly different from the control group (treatment A) at the $5 \%$ level.

* Significantly different from the control group (treatment A) at the I \% level. 
significantly with increasing vitamin $\mathrm{A}$ in the diet and this was associated with significant falls in the concentration of both $\alpha$-tocopherol and ubiquinone.

Table 5 shows that tocopherol increased the concentration of ubiquinone in the uterus, irrespective of vitamin A supplementation. Vitamin A supplementation had no effect on either tocopherol or ubiquinone concentration in this tissue. Only two results

Table 7. Expt r. Effect of two levels of vitamin $A$ supplementation on tissue concentrations of ubiquinone, $\alpha$-tocopherol and vitamin $A$ in the rat

(Each value is the mean for the results at each dietary level of vitamin A given in Tables $1-5$, regardless of treatment $A-G$ )

\begin{tabular}{|c|c|c|c|c|}
\hline \multirow[b]{2}{*}{ 'Tissue } & \multirow[b]{2}{*}{ Parameter } & \multicolumn{3}{|c|}{ Diet } \\
\hline & & Ao & Aro & $\mathrm{A}_{40}$ \\
\hline Heart & $\begin{array}{l}\text { Ubiquinone }(\mu \mathrm{g} / \mathrm{g}) \\
\alpha \text {-Tocopherol }(\mu \mathrm{g} / \mathrm{g}) \\
\text { Vitamin A (i.u. } / \mathrm{g})\end{array}$ & $\begin{array}{r}168 \\
14.7 \\
3.2\end{array}$ & $\begin{array}{l}I 64 \\
I I \cdot I * * * \\
3 \cdot 2\end{array}$ & $\begin{array}{l}\text { I5 } \\
9.4 * * \\
3.5\end{array}$ \\
\hline Liver & $\begin{array}{l}\text { Ubiquinone }(\mu \mathrm{g} / \mathrm{g}) \\
\alpha \text {-Tocopherol }(\mu \mathrm{g} / \mathrm{g}) \\
\text { Vitamin A (i.u./g) }\end{array}$ & $\begin{array}{l}107 \\
14 \cdot 1 \\
15\end{array}$ & $\begin{array}{r}74^{* *} \\
13 \cdot 2 \\
214^{* *}\end{array}$ & $\begin{array}{c}75^{* *} \\
13 \cdot 6 \\
259^{* *}\end{array}$ \\
\hline Kidney & $\begin{array}{l}\text { Ubiquinone }(\mu \mathrm{g} / \mathrm{g}) \\
\alpha \text {-Tocopherol }(\mu \mathrm{g} / \mathrm{g}) \\
\text { Vitamin A (i.u. } / \mathrm{g})\end{array}$ & $\begin{array}{l}82 \\
8 \cdot 9 \\
3 \cdot 8\end{array}$ & $\begin{array}{l}83 \\
8 \cdot 8 \\
3 \cdot 7\end{array}$ & $\begin{array}{c}6 I^{*} * \\
6 \cdot 5 \\
3 \cdot 8\end{array}$ \\
\hline Lung & $\begin{array}{l}\text { Ubiquinone }(\mu \mathrm{g} / \mathrm{g}) \\
\alpha-\text { Tocopherol }(\mu \mathrm{g} / \mathrm{g}) \\
\text { Vitamin A (i.u. } / \mathrm{g})\end{array}$ & $\begin{array}{l}15 \\
13 \cdot 1 \\
4 \cdot 4\end{array}$ & $\begin{array}{l}12^{*} \\
10 \cdot 0^{*} \\
6 \cdot 8^{*}\end{array}$ & $\begin{array}{l}\mathrm{I} 3^{*} \\
9^{*} 2^{*} \\
\mathrm{II} \cdot 4^{* *}\end{array}$ \\
\hline Uterus & $\begin{array}{l}\text { Ubiquinone }(\mu \mathrm{g} / \mathrm{g}) \\
\alpha \text {-Tocopherol }(\mu \mathrm{g} / \mathrm{g})\end{array}$ & $\begin{array}{l}15 \\
22 \cdot 4\end{array}$ & $\begin{array}{l}14 \\
24 \cdot 2\end{array}$ & $\begin{array}{l}16 \\
20 \cdot 7\end{array}$ \\
\hline
\end{tabular}

* Significantly different from the control group (diet Ao) at the $5 \%$ level.

** Significantly different from the control group (diet Ao) at the I \% level.

Table 8. Expt $\mathrm{I}$. Effect of vitamin E, Santoquin and selenium on concentrations of ascorbic acid and peroxides in livers and kidneys of rats

(Groups $1-7$ were on $\operatorname{diet}$ AO, 8-14 on $\operatorname{diet} A$ IO and $15^{-2}$ I on $\operatorname{diet} \mathrm{A}_{40}$, see p. 426)

\begin{tabular}{|c|c|c|c|c|c|c|c|}
\hline \multirow{2}{*}{$\begin{array}{c}\text { Group } \\
\text { nos. }\end{array}$} & \multirow{2}{*}{ Treatment } & \multicolumn{2}{|c|}{$\begin{array}{c}\begin{array}{c}\text { Ascorbic acid } \\
(\mu \mathrm{g} / \mathrm{g})\end{array}\end{array}$} & \multicolumn{2}{|c|}{$\begin{array}{c}\text { In vivo } \\
\text { peroxides } \uparrow \\
(\mu \mathrm{g} / \mathrm{g})\end{array}$} & \multicolumn{2}{|c|}{$\begin{array}{l}\text { Peroxide } \\
\text { production } \dagger\end{array}$} \\
\hline & & Liver & Kidney & Liver & Kidney & Liver & Kidney \\
\hline $\mathbf{I}, 8, \mathbf{I}_{5}$ & A. None & I6r & 79 & $8 \cdot 7$ & 4 & I 5 & 8 \\
\hline $2,9,16$ & B. Santoquin, final dose & 239 & 107 & $9 \cdot 5$ & 5 & I5 & 9 \\
\hline $3,10,17$ & C. $\alpha$-Tocopherol, final dose & 206 & IOO & $3.5 *$ & 2 & $7^{*}$ & 6 \\
\hline $4, I I, I 8$ & D. Santoquin, $0.015 \%$ of diet & 205 & 87 & $1 \mathrm{I} \cdot 3^{* * * *}$ & 4 & $2 \mathrm{I}$ & 10 \\
\hline $5, \mathbf{2}, \mathbf{1 9}$ & E. Santoquin, $0.10 \%$ of diet & 231 & 96 & $6 \cdot 0^{*}$ & 4 & IO*** & 10 \\
\hline $6,13,20$ & F. $\alpha$-Tocopherol, o.o I $\%$ of diet & 218 & 87 & $2 \cdot 7^{*}$ & 3 & $3 *$ & 7 \\
\hline $7,14,21$ & G. Se, 0.1 p.p.m. in diet & 197 & 92 & 10.0 & 4 & 17 & 9 \\
\hline
\end{tabular}

One rat was taken at random from each of the three groups that received the same treatment (A-G). Each value in the table is the mean of these three separate determinations.

* Significantly lower than control at $P=0.01$.

** Significantly lower than control at $P=0.05$.

*** Significantly higher than control at $P=0.05$.

+ Results in each column are optical densities at $\lambda_{\max } 520 \mathrm{~m} \mu$ (see p. 426). 
unfortunately were obtained for the higher dietary level of Santoquin and a significant effect for this substance could not be established. However, it did increase ubiquinone concentration in some groups, but not in others: this increase was always associated with an increase in tocopherol content.

Table 8 gives the results for ascorbic-acid concentrations, 'in vivo' peroxide concentrations and 'in vitro' peroxidation produced under standard conditions in the livers and kidneys of single animals taken from nineteen of the twenty-one groups. Santoquin, $\alpha$-tocopherol and Se all raised ascorbic-acid concentrations over those of the controls but these increases were not statistically significant. 'In vivo' peroxides and 'in vitro' peroxidation in liver were significantly reduced by $\alpha$-tocopherol and by the higher level of Santoquin in the diet. For kidney no significant differences emerged.

Expt 2. Diet SAFE, which contained no detectable amount of Se or vitamin A, no added fat and hardly any tocopherol, did not produce rats with tissues low in vitamin $\mathrm{E}$,

Table 9. Expt 2. Effect of vitamin E, vitamin $A$ and selenium on ubiquinone and ubichromenol concentrations in the hearts of very young rats on diet SAFE (see p. 426)

\begin{tabular}{|c|c|c|c|c|c|c|}
\hline $\begin{array}{l}\text { Group } \\
\text { no. }\end{array}$ & Survivors & s Treatment & $\begin{array}{l}\text { Mean heart } \\
\text { weight }(\mathrm{g})\end{array}$ & $\begin{array}{l}\text { Ubiquinone } \\
\qquad(\mu \mathrm{g} / \mathrm{g})\end{array}$ & $\begin{array}{l}\text { Ubichromenol } \\
(\mu \mathrm{g} / \mathrm{g})\end{array}$ & $\begin{array}{c}\alpha \text {-Tocopherol } \\
(\mu \mathrm{g} / \mathrm{g})\end{array}$ \\
\hline $\mathbf{I}$ & I I / I 6 & None & $0 \cdot 353$ & 88 & 7 & $10^{\circ} 0$ \\
\hline 2 & II $/ 14$ & $\begin{array}{l}\alpha-\text {-Tocopherol, } \\
\text { final dose }\end{array}$ & 0.385 & 106 & 8 & 19.7 \\
\hline 3 & II $/ \mathrm{I} 6$ & $\begin{array}{l}\text { Vitamin } A, \\
\text { final dose }\end{array}$ & 0.362 & 85 & 7 & $10 \cdot 0$ \\
\hline 4 & $10 / x_{5}$ & Se, final dose & 0.410 & 95 & 7 & 10.3 \\
\hline 5 & $13 / 16$ & $\begin{array}{l}\text { Vitamin } A \text {, } \\
40 \text { i.u. } / g \text { of diet }\end{array}$ & $0.3^{81}$ & $9 I$ & 7 & $8 \cdot 4$ \\
\hline 6 & $8 / 14$ & $\begin{array}{l}\alpha \text {-Tocopherol, } \\
0.01 \% \text { of diet }\end{array}$ & 0.403 & 125 & Io & $\mathbf{I} 7 \cdot \mathbf{I}$ \\
\hline 7 & $14 / 15$ & None & 0.347 & I I4 & 7 & $5 \cdot 9$ \\
\hline 8 & $8 / 14$ & $\begin{array}{l}\alpha \text {-Tocopherol, } \\
\text { final dose }\end{array}$ & 0.400 & I I 5 & 8 & 10.4 \\
\hline 9 & $10 / 14$ & $\begin{array}{l}\text { Vitamin A, } \\
\text { final dose }\end{array}$ & 0.334 & 110 & 8 & $5 \cdot 4$ \\
\hline 10 & I I $/ \mathrm{I} 6$ & $\begin{array}{l}\text { Vitamin A, } \\
40 \text { i.u./g of diet }\end{array}$ & 0.384 & 118 & 7 & $5^{\cdot} 9$ \\
\hline I I & $9 / 15$ & $\begin{array}{l}\alpha \text {-Tocopherol, } \\
0.01 \% \text { of diet }\end{array}$ & 0.360 & 108 & 3 & $15 \cdot 5$ \\
\hline
\end{tabular}

as had been hoped: the reserves of young animals born from normal dams are obviously too high to be depleted in 3 weeks even on this diet. The results of the experiments on these young rats (Tables 9 and 10 ) completely confirmed the previous findings. Dosing with $\alpha$-tocopherol increased ubiquinone concentrations but dosing with vitamin A (group 3) depressed tocopherol and ubiquinone concentrations in the liver. For the first time (group 4) a single dose of selenite was given, and it raised the ubiquinone concentration, particularly in liver, and nearly doubled that of ubichromenol in it. Supplements of vitamin A in the diet had no effect (group 5) compared with those of vitamin E (group 6). Se in the diet (group 7) again produced a pronounced effect on ubiquinone concentration, particularly in liver; when animals receiving Se (groups 8 and II) were given $\alpha$-tocopherol, no further increase was produced, which confirmed the essential similarity in the effects of the two nutrients. 
Vitamin A (groups 9 and ro) given to animals receiving Se had little effect on the ubiquinone content in the heart, but, as usual, significantly depressed that in the liver.

Expt 3. In this test (Table I I), Santoquin and DPPD were studied together. Both anti-oxidants clearly reached the tissues. They exhibited their usual protective effect on vitamin A (increased liver storage), and significantly protected against the production of peroxides in liver. Neither substance, however, was able to increase the ubiquinone content of heart or liver.

Table 10. Expt 2. Effect of vitamin $E$, vitamin $A$ and selenium on ubiquinone and ubichromenol concentrations in the livers of very young rats on diet SAFE (see p. 426)

\begin{tabular}{|c|c|c|c|c|c|}
\hline $\begin{array}{l}\text { Group } \\
\text { no. }\end{array}$ & Treatment & $\begin{array}{c}\text { Mean liver } \\
\text { weight }(g)\end{array}$ & $\begin{array}{c}\text { Ubiquinone } \\
(\mu \mathrm{g} / \mathrm{g})\end{array}$ & $\begin{array}{l}\text { Ubichromenol } \\
\qquad(\mu \mathrm{g} / \mathrm{g})\end{array}$ & $\begin{array}{c}\alpha-\text { Tocopherol } \\
(\mu \mathrm{g} / \mathrm{g})\end{array}$ \\
\hline I & None & $3 \cdot 63$ & 93 & 20 & $3 \cdot 7$ \\
\hline 2 & $\alpha$-Tocopherol, final dose & 3.55 & 105 & 20 & $9 \cdot 7$ \\
\hline 3 & Vitamin $A$, final dose & $3 \cdot 83$ & 87 & 20 & $2 \cdot 9$ \\
\hline 4 & Se, final dose & $3 \cdot 81$ & 121 & 38 & $3 \cdot 4$ \\
\hline 5 & $\begin{array}{l}\text { Vitamin A, } \\
40 \text { i.u./g of diet }\end{array}$ & $4^{1} 16$ & 100 & 20 & $6 \cdot 4^{*}$ \\
\hline 6 & $\begin{array}{l}\alpha \text {-Tocopherol, } \\
\text { o.or \% of diet }\end{array}$ & $4 \cdot 14$ & I 59 & 27 & $16 \cdot 7$ \\
\hline 7 & None & $3 \cdot 49$ & I 74 & 25 & $\mathrm{I} \cdot 5$ \\
\hline 8 & $\alpha$-Tocopherol, final dose & 3.84 & 154 & 25 & $I I \cdot 2$ \\
\hline 9 & Vitamin $A$, final dose & 3.54 & II 2 & 26 & $6 \cdot 5^{*}$ \\
\hline Io & $\begin{array}{l}\text { Vitamin A, } \\
40 \text { i.u./g of diet }\end{array}$ & 4.70 & I 14 & $2 \mathrm{I}$ & $9 \cdot 3 *$ \\
\hline I I & $\begin{array}{l}\alpha \text {-Tocopherol, } \\
0.01 \% \text { of diet }\end{array}$ & $3 \cdot 46$ & 163 & 32 & $I 6 \cdot I$ \\
\hline
\end{tabular}

Groups 7-i I all received a dietary supplement of 0.1 p.p.m. of Se.

* Spuriously high, owing to presence of vitamin A decomposition product in spot extract: reading at $520 \mathrm{~m} \mu$ continued to increase after $2 \mathrm{~min}$.

Table I I. Expt 3. Effect of Santoquin and DPPD on vitamin $A$ and ubiquinone concentrations in rats and their protective action on peroxide formation (see p. 427)

\begin{tabular}{|c|c|c|c|c|c|c|c|c|}
\hline Treatment & Organ & $\begin{array}{l}\text { Ubi- } \\
\text { quinone } \\
(\mu \mathrm{g} / \mathrm{g})\end{array}$ & $\begin{array}{c}\text { Ubi- } \\
\text { chromenol } \\
(\mu \mathrm{g} / \mathrm{g})\end{array}$ & $\begin{array}{c}\alpha-\text { Toco- } \\
\text { pherol } \\
(\mu \mathrm{g} / \mathrm{g})\end{array}$ & $\begin{array}{c}\text { Vitamin A } \\
\text { (i.u./g) }\end{array}$ & $\begin{array}{l}\text { pe } \\
(E \\
(\mathrm{I})\end{array}$ & $\begin{array}{l}\text { Live } \\
\text { roxi } \\
\text { at } 52 \\
(2)\end{array}$ & $\begin{array}{l}\text { r } \\
\operatorname{des} \\
o m \mu) \\
(3)\end{array}$ \\
\hline None & $\begin{array}{l}\text { Heart } \\
\text { Liver }\end{array}$ & $\begin{array}{l}208 \\
106\end{array}$ & $\begin{array}{l}21 \\
52\end{array}$ & $\begin{array}{l}3 \cdot 6 \\
2 \cdot 5\end{array}$ & I 16 & 4 & I I & 14 \\
\hline $0.05 \%$ DPPD & $\begin{array}{l}\text { Heart } \\
\text { Liver }\end{array}$ & $\begin{array}{r}186 \\
96\end{array}$ & $\begin{array}{l}\text { I3 } \\
78\end{array}$ & $\begin{array}{l}4 \cdot 0 \\
2 \cdot 3\end{array}$ & r 73 & 3 & 6 & $8^{*}$ \\
\hline $0.10 \%$ Santoquin & $\begin{array}{l}\text { Heart } \\
\text { Liver }\end{array}$ & $\begin{array}{l}227 \\
114\end{array}$ & $\begin{array}{l}16 \\
59\end{array}$ & $\begin{array}{l}4 \cdot 7 \\
2 \cdot 3\end{array}$ & 146 & 3 & 7 & II* \\
\hline
\end{tabular}

* Significantly different from controls $(P=0.01)$.

$\uparrow$ Each result is a mean of three determinations at (I) zero time, (2) ro min and (3) after $15 \mathrm{~min}$ incubation at $37^{\circ}$.

Expt 4. Table I2 shows that when O.I p.p.m. Se was added to the diet of stockcolony rats for 7 weeks, ubiquinone and ubichromenol concentrations were increased in the heart.

Expt 5. In the previous studies on the uterus, the tissue taken for analysis usually 
included part of the fallopian tube and ovary. In this experiment the uterus and ovary were studied separately (Table 13 ). A clear-cut difference in response between the two tissues was found. Se, which did not affect that in the uterus (thus confirming the results of Expt I), significantly increased the ubiquinone content of the ovary. Tocopherol increased ubiquinone concentrations in both tissues. The ovary, even in the deficient animals, contained much tocopherol and, further, exhibited a remarkable increase in tocopherol concentration when the rats were dosed with it, even more so than the uterus. In this experiment, the pituitary was also studied, since Quaife, Swanson, Dju \& Harris (1949) have claimed that this organ contained goo $\mu \mathrm{g}$ (sic) tocopherol/g fresh tissue. We found no evidence that pituitary tissue took up much tocopherol from the dose given, nor could ubiquinone be demonstrated in this organ.

Table I2. Expt 4. Effect of selenium on ubiquinone and ubichromenol concentration in the hearts and livers of rats on the stock diet $\left(S D_{\mathrm{I}}\right)$

(Mean values for groups of six female rats)

\begin{tabular}{llccc} 
Organ & \multicolumn{1}{c}{ Treatment } & $\begin{array}{c}\text { Ubiquinone } \\
(\mu \mathrm{g} / \mathrm{g})\end{array}$ & $\begin{array}{c}\text { Ubichromenol } \\
(\mu \mathrm{g} / \mathrm{g})\end{array}$ & $\begin{array}{c}\alpha \text {-Tocopherol } \\
(\mu \mathrm{g} / \mathrm{g})\end{array}$ \\
Heart & None & 102 & 8 & 21 \\
& Supplement of Se & 163 & 12 & 17 \\
\multirow{2}{*}{ Liver } & None & 109 & 64 & 29 \\
& Supplement of Se & 106 & 64 & 30
\end{tabular}

Table ${ }_{13}$. Expt 5. Effect of vitamin $E$ and selenium on ubiquinone and ubichromenol concentration in rat ovary, uterus and pituitary

(Mean values for groups of ten female rats)

\begin{tabular}{|c|c|c|c|c|c|}
\hline Organ & Treatment & $\begin{array}{l}\text { Mean organ } \\
\text { weight }(g)\end{array}$ & $\begin{array}{l}\text { Ubiquinone } \\
(\mu \mathrm{g} / \mathrm{g})\end{array}$ & $\begin{array}{l}\text { Ubichromenol } \\
(\mu \mathrm{g} / \mathrm{g})\end{array}$ & $\begin{array}{c}\alpha \text {-Tocopherol } \\
(\mu \mathrm{g} / \mathrm{g})\end{array}$ \\
\hline Ovary & $\begin{array}{l}\text { Vitamin E deficiency } \\
\alpha \text {-Tocopherol, final dose } \\
\text { Se, final dose }\end{array}$ & $\begin{array}{l}0.039 \\
0.038 \\
0.050\end{array}$ & $\begin{array}{l}23 \\
42 \\
38\end{array}$ & $\begin{array}{l}\text { ND } \\
\text { ND } \\
\text { ND }\end{array}$ & $\begin{array}{l}34 \cdot 6 \\
120 \\
4 r \cdot 0\end{array}$ \\
\hline Uterus & $\begin{array}{l}\text { Vitamin E deficiency } \\
\alpha \text {-Tocopherol, final dose } \\
\text { Se, final dose }\end{array}$ & $\begin{array}{l}0.396 \\
0.373 \\
0.320\end{array}$ & $\begin{array}{l}3 \\
9 \\
3\end{array}$ & $\begin{array}{l}2 \\
3 \\
1\end{array}$ & $\begin{array}{r}6 \cdot 2 \\
24 \cdot 8 \\
6 \cdot 6\end{array}$ \\
\hline Pituitary & $\begin{array}{l}\text { Vitamin E deficiency } \\
\alpha \text {-Tocopherol, final dose }\end{array}$ & $\begin{array}{l}0.067 \\
0.077\end{array}$ & $\begin{array}{l}\text { ND } \\
\text { ND }\end{array}$ & $\begin{array}{l}\text { ND } \\
\text { ND }\end{array}$ & $\begin{array}{l}11 \cdot 4 \\
12 \cdot 9\end{array}$ \\
\hline
\end{tabular}

\section{DISCUSSION}

The experiments reported here have, in general, confirmed previous findings. It now seems reasonably clear that the effect of vitamin $E$ on ubiquinone concentrations in the rat is not appreciably influenced by the level of vitamin A in the diet. As suggested by Edwin et al. (1961), the effect of vitamin A seems to be almost entirely manifested through its depressant action on vitamin $\mathrm{E}$ concentration in tissues other than liver. However, our experiments lend some support to an earlier suggestion that vitamin A probably has a more direct effect on ubiquinone concentration in liver, besides the effect through vitamin $\mathrm{E}$. It is apparent also that the effect of vitamin $\mathrm{E}$ is 
not limited to tissues with a low initial concentration of tocopherol. For example, the hearts of the very young rats in Expt 2 had a high concentration of tocopherol, but dosing with vitamin $\mathrm{E}$ still exerted a marked effect on ubiquinone contents.

It is now recognized that $\mathrm{Se}$ and $\alpha$-tocopherol have closely similar physiological effects on certain signs of vitamin E deficiency. However, on certain others Se appears to have no effect. This remarkable relationship between two such dissimilar substances presents a problem that must be solved if a deeper understanding of them is to be achieved. It is possible, perhaps somewhat arbitrarily, to distinguish four main types of the vitamin E-deficiency syndrome. They are:

(I) The steatopathies, which include the 'yellow fat' diseases of species such as mink, fox, ferret, cat and pig. In all probability they are the least complicated conditions and may be a more or less simple result of a dearth of anti-oxidants, leading to lipid peroxidation readily prevented by a number of anti-oxidants besides vitamin $\mathrm{E}$. There has so far been no report of the prevention by Se of any of these diseases. Søndergaard, Christensen, Dam \& Prange (1958) have shown that Se does not prevent the peroxidation of body fat in the rat.

(2) The liver diseases, which include necrotic liver degeneration in the mouse and rat and hepatosis dietetica in the pig, constitute the second group. These conditions are produced only by a combined deficiency of Se and $\alpha$-tocopherol and can be prevented by either.

(3) A third group of syndromes, although apparently unrelated physiologically and diverse in their nature, are apparently caused by combined deficiency of Se and vitamin $\mathrm{E}$ and can usually be completely prevented by the presence of one of these nutrients, but only partially by the other. In several diseases of this group, lipid peroxidation may play a subsidiary part, and they can often be exacerbated by the presence of unsaturated fat in the diet. These diseases cover a range in which the requirements for Se and $\alpha$-tocopherol appear to be mutually dependent on each other, the complete picture being in general still obscure. On the one extreme there is the exudative diathesis of chicks, which responds completely to $\alpha$-tocopherol but, according to Nesheim \& Scott (1958), not quite completely to Se. These authors opine that both nutrients may be necessary for the chick, the tocopherol in minimal amounts. At the other extreme there is 'stiff lamb' disease, which is prevented almost entirely by Se, but with such difficulty by $\alpha$-tocopherol that there is some doubt as to whether it is a vitamin E-deficiency state (cf. Muth, Oldfield, Schubert \& Remmert, 1959; Oldfield, Muth \& Schubert, 1960). Between these two extremes lie the degenerative diseases of muscle in chicks and cattle, and probably other conditions, against which only one of the two nutrients is an effective protective agent, the other being partially so. Thus Dam \& Søndergaard (1957) could not completely prevent muscle degeneration in chicks with Se, whereas nutritional muscular dystrophy in calves responds to vitamin E (Blaxter \& Sharman, I953), but variably to Se (cf. Sharman, Blaxter \& Wilson, 1959; Maplesden \& Loosli, 1960). It seems that the naturally occurring dystrophies in both lambs and calves may be primarily Se deficiency, whereas the dystrophies induced in these animals by cod-liver oil are mainly vitamin $\mathrm{E}$ deficiency (in this connexion, see also Welch, Hoekstra, Pope \& Phillips, 1960). 
(4) A group of diseases apparently preventable only by vitamin E. It includes many (and perhaps all) conditions affecting reproduction and embryonic development, the testicular degenerations, as well as several other unrelated conditions, such as encephalomalacia in the chick, muscular dystrophies in the rabbit, rat and pig, and diminished resistance to haemolysis of erythrocytes in several species. Se has been shown to be ineffective in preventing resorption of the foetus in the rat (Harris et al. 1958), loss of both fertility and hatchability in the turkey (Creger, Mitchell, Atkinson, Ferguson, Reid \& Couch, 1960), loss of hatchability and infant mortality in poultry (Jensen \& McGinnis, 1960), muscular dystrophy in the rabbit (Hove, Fry \& Schwarz, 1958; Draper, 1957), muscular dystrophy in the guinea-pig (Seidel \& Harper, 1960), encephalomalacia in the chick (Dam, Nielsen, Prange \& Søndergaard, 1957) and erythrocyte haemolysis in the rat (Søndergaard et al. 1958).

In this context, the finding that Se is without effect on the ubiquinone concentration in rat uterus, although it is active in other tissues, assumes some significance. Green, Diplock, Bunyan \& Edwin (1961) have suggested that syndromes of vitamin E deficiency may be of a local character and may be caused by a lowered concentration of $\alpha$-tocopherol in a particularly susceptible tissue. In the rat, such a tissue is the uterus. Although tocopherol increases ubiquinone concentration in the uterus, this function might be regarded as adventitious in this tissue, and the function of the tocopherol in the uterus may be mainly to satisfy the demands of prospective embryos. On this view, therefore, the failure of Se to have an effect on ubiquinone concentration in the uterus may be epiphenomenal and not directly the reason why it fails to prevent resorption of the foetus. Rather it may be diagnostic evidence that $\mathrm{Se}$, in whatever active form is necessary, either may not reach the uterus at all or, if it does, that the process of ubiquinone biogenesis in the foetus cannot be influenced by it. As a corollary, the marked effect of Se in increasing ubiquinone concentration in the liver may be linked to its facile role in preventing the deficiency diseases of the liver. Further studies are necessary before this hypothesis can be accepted, but the evidence from the rat supports the idea that, though both Se and $\alpha$-tocopherol may play a part in ubiquinone synthesis, they are not equally effective in different tissues.

Although it is clearly evident from our experiments (and from some described previously by Edwin et al. 1961) that Se can increase ubiquinone concentration in the livers of rats on a typical vitamin E-deficient diet, i.e. a diet that, though producing gestation-resorption, contains enough Se to prevent the onset of hepatic necrosis, it was, even so, surprising to find in Expt 4 that Se could also increase ubiquinone concentration in rats on the stock diet. It would certainly seem that this so-called adequate diet was in fact not really adequate in either tocopherol or Se, if the biochemical index of increase in ubiquinone concentration can be taken as having some nutritional significance, for it is apparent from Expt 3 that $\alpha$-tocopherol cannot increase ubiquinone concentrations in animals receiving adequate Se (compare groups 7, 8 and II) and that, similarly, Se has no effect if the tocopherol content is adequate (compare groups 6 and Ir). This effect indeed suggests an adverse biochemical evaluation of diets judged adequate by normal nutritional standards.

These experiments seem to confirm our previous contention that the function of 
$\alpha$-tocopherol and some other substances as 'in vivo' anti-oxidants can now be separated from the specific role of $\alpha$-tocopherol. They show that levels of Santoquin and DPPD that can effectively fulfil an anti-oxidant function, that is, can protect vitamin A, reduce 'in vivo' peroxides and reduce 'in vitro' peroxidation, are incapable of influencing ubiquinone content as does $\alpha$-tocopherol (with the possible exception of a slight effect of Santoquin on the uterus). Se, on the other hand, increases ubiquinone concentrations but does not protect against tissue peroxidation in the rat (cf. Bieri (I959), who found that Se protected chick tissues; but peroxidation is much less in the chick than in the rat). It is difficult to reconcile our present findings with the anti-oxidant theory. For example, they might be explained by the hypothesis that $\alpha$-tocopherol can perform its anti-oxidant function in some system that is denied to substances such as Santoquin and DPPD and that this system, sensitive to oxidation in the absence of tocopherol, is part of a mechanism for ubiquinone synthesis. How then does Se function? It does not seem likely that this element given at less than one-hundredth of the molar concentration of tocopherol could be as efficient an in vivo anti-oxidant as the latter. No derivative of Se with such properties is known, and Se added to vitamin E-deficient diets does not have any influence on tissue peroxidation. Further results (being prepared for publication) have also shown that Se is without such activity in rats when added to a so-called necrogenic (i.e. Sedeficient) diet in amounts sufficient to prevent liver necrosis. Although Tappel, Zalkin \& Knapp ( 1960 ) have stated that 'anti-oxygenic' compounds formed in vivo account for the action of Se in vitamin E-deficiency conditions in the chick, the only substance of such a type that we have observed to increase in amount in the rat after a Se supplement is ubichromenol. Unpublished observations in this laboratory have indicated, however, that ubichromenol 50 has only about one-tenth of the activity of $\alpha$-tocopherol in preventing lipid peroxidation in vitro.

It would seem that $\alpha$-tocopherol has functions of two types. First, it can act as a tissue anti-oxidant, and this role can in certain circumstances be taken over by other substances, such as the synthetic anti-oxidants DPPD and Santoquin, to different degrees in different tissues. These anti-oxidants probably exert their effects both by sparing vitamin $\mathrm{E}$ (see Tables $\mathrm{I}-3$ ) and also by a true protective action on lipid peroxidation, which is often involved in vitamin E-deficiency conditions, even when the site of the lesion is muscular or vascular (see Dam, 1957). Secondly, in the rat and the rabbit, $\alpha$-tocopherol has a more specific role, which is in some way concerned with the regulation of ubiquinone concentrations and is distinct from its anti-oxidant function. The mechanism by which the regulation takes place is so far unknown. It could be a direct effect at some stage of synthesis or an indirect effect through other systems. It is not yet known how far the relation between vitamin $\mathrm{E}$ and ubiquinone can be extended to other species. Nor is it yet known whether these two functions of vitamin $\mathrm{E}$ are the only ones. It is therefore still necessary to proceed with caution before their significance in explaining the total biological role of vitamin $\mathrm{E}$ can be assessed. 


\section{SUMMARY}

I. The influence of vitamin $A$ status on the relation between vitamin $E$ and ubiquinone has been studied in groups of vitamin E-deficient female rats. The previously observed effect of vitamin $E$ in increasing ubiquinone content in the tissues of these rats was not influenced by varying the vitamin $A$ content of the diet.

2. Rat ovary was found to have a high concentration of $\alpha$-tocopherol. The level of ubiquinone in the ovary could be markedly increased when either $\alpha$-tocopherol or selenium was administered to vitamin E-deficient rats. Pituitary glands, contrary to expectation, proved to contain little tocopherol or ubiquinone.

3. Se, administered to vitamin E-deficient rats, significantly enhanced ubiquinone concentrations in all tissues examined except the uterus. The same effect was observed whether $0 . \mathrm{I}$ p.p.m. was given in the diet or $2-10 \mu \mathrm{g}$ as a single dose. The relation between the failure of Se to influence ubiquinone concentration in the uterus and the reported failure of this substance to prevent resorption of the foetus in the rat is discussed.

4. Although the influence of Se and $\alpha$-tocopherol on ubiquinone concentrations is complementary, small amounts of Se raised ubiquinone concentrations even in rats on the stock-colony diet. It would appear that this diet may not contain an optimal amount of either nutrient.

5. Two powerful anti-oxidants, Santoquin and DPPD, which like $\alpha$-tocopherol were able to protect vitamin $\mathrm{A}$ and reduce lipid peroxidation in liver, were without significant influence on ubiquinone concentrations in the rat. Se, on the other hand, showed no anti-oxidant activity at all and yet had a marked influence on ubiquinone concentrations. On the basis of these experiments, it is suggested that vitamin $\mathrm{E}$ and Se have a specific biological role distinct from the general anti-oxidant function of the former. This role would appear to relate in some way, at present unknown, to control over concentrations of ubiquinone.

\section{REFERENCES}

Bieri, J. G. (1959). Nature, Lond., 184, I 148.

Blaxter, K. L. \& Sharman, G. A. M. (1953). Nature, Lond., 172, 1006.

Creger, C. R., Mitchell, R. H., Atkinson, R. L., Ferguson, T. M., Reid, B. L. \& Couch, J. R. (1960). Poult. Sci. 39, 59.

Dam, A. (1957). Pharmacol. Rev. 9, I.

Dam, H., Nielsen, G. K., Prange, I. \& Søndergaard, E. (1957). Experientia, 13, 493.

Dam, H. \& Søndergaard, E. (1957). Experientia, r3, 494 .

Diplock, A. T., Green, J., Edwin, E. E. \& Bunyan, J. (196o). Biochem. 7. 76, 563 .

Draper, H. H. (1957). Nature, Lond., 180, I419.

Draper, H. H., Goodyear, S., Barbee, K. D. \& Johnson, B. C. (1958). Brit. F. Nutr. 12, 89.

Edwin, E. E., Diplock, A. T., Bunyan, J. \& Green, J. (196r). Biochem. F. 79, 9 I.

Geschwind, I. I., Williams, B. S., \& Li, C. H. (195 I). Acta Endocrinol. 8, 247.

Green, J., Diplock, A. T., Bunyan, J. \& Edwin, E. E. (1961). Biochem. F. 79, 108.

Green, J., Edwin, E. E., Diplock, A. T. \& Bunyan, J. (1960). Biochem. Biophys. Res. Commun. $2,388$.

Harris, P. L., Ludwig, M. I. \& Schwarz, K. (1958). Proc. Soc. exp. Biol., N.Y., 97, 686.

Heaton, F. W., Lowe, J. S. \& Morton, R. A. (1957). Biochem. F. 67, 208.

Hove, E. L., Fry, G. S. \& Schwarz, K. (1958). Proc. Soc. exp. Biol., N. Y., 98, 27.

Jensen, L. S. \& McGinnis, J. (1960). F. Nutr. 72, 23.

McCay, P. B., Carpenter, M. P., Kitabchi, A. E. \& Caputto, R. (1959). Arch. Biochem. Biophys. 82, 472.

Maplesden, D. C. \& Loosli, J. K. (1960). F. Dairy Sci. 43, 645 . 
Moore, T. \& Sharman, I. M. (1960). Brit. F. Nutr. I4, 473.

Muth, O. H., Oldfield, J. E., Schubert, J. R. \& Remmert, L. F. (1959). Amer. F. vet. Res. 20,23 I. Nesheim, M. C. \& Scott, M. L. (1958). F. Nutr. 65, 601.

Oldfield, J. E., Muth, O. H. \& Schubert, J. R. (1960). Proc. Soc. exp. Biol., N. Y., 103, 799.

Quaife, M. L., Swanson, W. J., Dju, M. Y. \& Harris, P. L. (1949). Ann. N. Y. Acad. Sci. 52, 300. Roe, J. H., Mills, M. B., Oesterling, M. J. \& Damron, C. M. (1948). F. biol. Chem. 174, 201.

Seidel, J. C. \& Harper, A. E. (1960). F. Nutr. 70, 147.

Sharman, G. A. M., Blaxter, K. L. \& Wilson, R. S. (1959). Vet. Rec. 71, 536.

Søndergaard, E., Christensen, F., Dam, H. \& Prange, I. (1958). Int. Abstr. biol. Sci., Suppl., p. 92. 'Tappel, A. L., Zalkin, H. \& Knapp, F. W. (1960). Fed. Proc. r9, 4 I9.

Welch, J. G., Hoekstra, W. G., Pope, A. L. \& Phillips, P. H. (1960). F. Anim. Sci. 19, 620.

Zalkin, H. \& Tappel, A. L. (1960). Arch. Biochem. Biophys. 88, I I 3. 